\title{
Re-visit local coupling correction in the interaction regions of $\mathrm{RHIC}$
}

\author{
Y. Luo, W. Fischer, C. Liu, A. Marusic, M. Minty, V. Ptitsyn, \\ V. Schoefer, S. Tepikian, D. Trbojevic, C. Zimmer
}

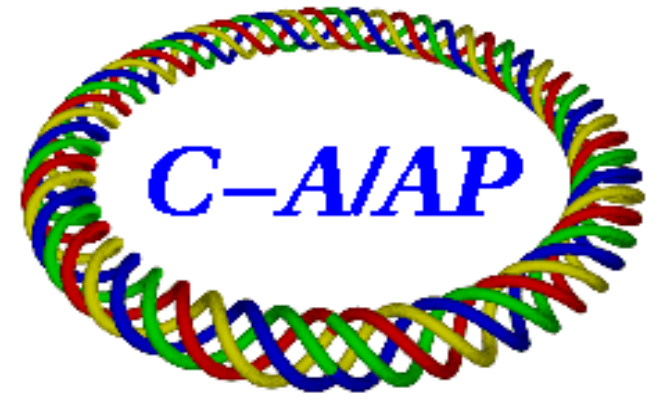

\section{Collider-Accelerator Department Brookhaven National Laboratory Upton, NY 11973}

Notice: This document has been authorized by employees of Brookhaven Science Associates, LLCunder Contract No. DE-AC02-98CH10886 with the U.S. Departm ent of En ergy. The United States Governm ent retains a nonexclusive, paid-up, irrevocable, world-wide license topublish or reproduce the published formof this document, or allow others to do so, for United States Government purposes. 


\title{
RE-VISIT LOCAL COUPLING CORRECTION IN THE INTERACTION REGIONS OF RHIC ${ }^{*}$
}

\author{
Y. Luo, W. Fischer, C. Liu, A. Marusic, M. Minty, V. Ptitsyn, V. Schoefer, \\ S. Tepikian, D. Trbojevic, C. Zimmer, Brookhaven National Laboratory, Upton, NY USA
}

Abstract

In this article we will re-visit the local coupling correction in the interaction regions (IRs) of the Relativistic Heavy Ion Collider (RHIC). We will review the measurement data of triplet quadrupole rolls, the local coupling correction strengths in the RHIC control system, and the methods for the local coupling correction with local skew quadrupole correctors. Based on the in-turnnel meaurement data of triplet roll errors in 2011, we will analytically calculate and simulate IR-bump method to find out the local skew correction strengths and compare them at store and at injection with the Blue and Yellow ring lattices in the 2011 polarized proton (p-p) and Au-Au runs. The vertical dispersion from the triplet roll errors, local and global coupling correction skew quadrupoles, and the vertical dipole correctors are calculated and discussed.

\section{INTRODUCTION}

The roll errors of normal quadruples and the vertical closed orbits in the normal sextupoles are the main sources of the betatron coupling in RHIC. There are 3 families of 48 skew quadrupoles for the global coupling correction. And there are 12 local skew quadrupole correctors in the 6 interaction regions for the local coupling correction. Global coupling correction aims at reducing the eignmode tune split and guarantees a robust tune feedback. The local coupling correction will reduce cross-talk of orbit and dispersion between the horizontal and vertical planes.

The global coupling is measured with the global coupling coefficient $C^{-}$, which is defined as

$C^{-}=\left|C^{-}\right| e^{i \chi}=\frac{1}{2 \pi} \int_{0}^{L} \sqrt{\beta_{x} \beta_{y}} k_{s} e^{i\left[\Phi_{x}-\Phi_{y}-2 \pi \Delta \cdot s / L\right]} d l$.

For the RHIC p-p runs, the minimum tune split or $\left|C^{-}\right|$ should be smaller than $10^{-4}$ to maintain the proton polarization. From Eq. (1), to correct the global coupling we only need two orthogonal skew quadrupoles or families. The local coupling can be measured with the eignmode projections in $\mathrm{x}$ and $\mathrm{y}$ planes. It also can be measured by other methods, like coupling resonance driving terms, action-angle jump and so on. The orbit leakage in one plane from a local orbit bump in another plane and the vertical dispersion can be used as the observables of local coupling.

\footnotetext{
* This work was supported by Brookhaven Science Associates, LLC under Contract No. DE-AC02-98CH10886 with the U.S. Department of Energy.
}

In RHIC, the global tune and coupling feedback had been succesffully demonstrated in 2006 and has been implemented in the routine RHIC operations since then $[1,2]$. With tune and coupling feedback, the coupling coefficient or $\left|\Delta Q_{\min }\right|$ is corrected to below $10^{-4}$. After global coupling is corrected, the local coupling may still exist. In the 2011 RHIC proton run, we measured the vertical dispersion with a maximum $0.5 \mathrm{~m}$. Simulations show that the global coupling correction could introduce the same order of magnitude of the measured vertical dispersion [3]. Therefore, the best approach to correct both the local and global coupling is to correct the local coupling with the local skew quadrupole correctors first, followed by the global coupling feedback with the global skew quadrupoles. The local coupling in RHIC is corrected with the local skew quadrupole correctors. Their strengths haven't been changed since 2004 , although $\beta^{*}$ functions at IPs have been continuously reduced in the past 6 years. In this article we will re-visit the local coupling correction and investigate their impact on vertical dispersion.

\section{MAGNET ROLL MEASUREMENTS}

The magnet roll errors of RHIC triplet quadrupoles were first measured in the RHIC tunnel in 2001. In 2004, the rolls of the triplets in Sector 5 were slightly adjusted to cancel their contributions to global coupling. Since then, there is no in-tunnel IR magnet roll measurement and adjustment in RHIC. Table 1 lists the triplet roll errors from the measurements in 2001 and after 2004 adjustment [4]. In Table 1, the measurements of the triplet roll errors were not available in every sectors since it was found with beam that in some sectors the required local coupling correction strengths were small and therefore the measurement was not performed there. The roll errors are in units of mrad. In the magnet measurement and alignment, the magnet rolls are always observed from the interaction points (IPs). And the roll angles in the counter-clockwise direction are defined positive, no matter in the outer ring or in the inner ring, and no matter in the Blue ring or in the Yellow ring.

To model these measurements of triples rolls in accelerator codes, we need to know the definitions of positive roll angles in these codes. As we know, a normal quadrupole will change to a skew quadrupole by a roll angle of $45^{\circ}$ in the direction from positive $\mathrm{x}$ axis to positive $\mathrm{y}$ axis. The new skew quadrupole strength $K_{1 S}=-K_{1}$, where $K_{1}$ is the strength of the un-rolled normal quadrupole. In SimTrack [5], we define a positive roll angle for magnets if the magnet is rolled in the direction from positive $\mathrm{x}$ axis to the positive y axis direction. In the following study, we will 
model these roll errors and do simulation calculation with SimTrack.

Table 1: Triplets roll errors [ mrad ] after 2004 adjustment

\begin{tabular}{lccc}
\hline \hline Sector & Triplet & Blue Ring & Yellow Ring \\
\hline 5 & Q1 & 1.35 & -4.91 \\
& Q2 & 1.33 & -2.35 \\
& Q3 & 1.37 & -2.15 \\
\hline 6 & Q1 & 1.59 & -0.55 \\
& Q2 & -1.63 & -0.10 \\
& Q3 & -3.69 & 1.00 \\
\hline 7 & Q1 & -0.89 & - \\
& Q2 & 1.23 & - \\
& Q3 & -1.32 & - \\
\hline 8 & Q1 & 4.67 & 1.02 \\
& Q2 & -2.10 & -1.89 \\
& Q3 & 0.17 & 1.37 \\
\hline 1 & Q1 & 4.72 & 6.97 \\
& Q2 & 2.40 & 2.01 \\
& Q3 & 1.38 & -0.70 \\
\hline 2 & Q1 & 1.26 & 3.27 \\
& Q2 & 3.21 & -1.65 \\
& Q3 & -0.87 & -0.69 \\
\hline \hline
\end{tabular}

\section{ONLINE CORRECTION STRENGTHS}

Here we list the strength settings for the local skew quadrupole correctors in the RHIC control system. The same correction strengths have been used at injection, at store in the p-p and Au-Au runs. The correction strengths of local skew quadrupole correctors were found with beam in 2001-2004. Adjustment of triplet rolls in sector 5 was done in 2004. After the adjustment, the correction strengths for the local coupling correctors bi5qs3 and yo5qs3 were close to zero. Since then, the same set of strengths of the local skew quadrupole correctors have been used at store, at injection, and for the p-p and Au-Au runs.

In Table 2, the strength signs of the local skew quadrupole correctors in the Blue ring are different from that in the RHIC control system. From the convention of RHIC's magnet polarity definition, a positive quadrupole rolls $45^{\circ}$ in clockwise direction will give a positive skew quadrupole. As mentioned above, according to the normal magnet strength definition as in MADX, it actually will give a skew quadrupole with a negative strength. In the Yellow ring, we still use the right-hand coordinate system, that is, the positive $\mathrm{x}$ axis points out, the positive $\mathrm{y}$ axis points down and the proton beam circulates counterclockwise. In Table 2, the strength signs of the local skew quadrupole correctors in the Yellow ring are the same as that in the control system.
Table 2: Online local skew quadruple strengths $\left[10^{-3} \mathrm{~m}^{-1}\right]$

\begin{tabular}{cc|cc}
\hline \hline \multicolumn{2}{c|}{ Blue ring } & \multicolumn{2}{c}{ Yellow ring } \\
Name & Strength & Name & Strength \\
\hline bi5qs3 & 0 & yo5qs3 & -0.26 \\
bo6qs3 & 0.1 & yi6qs3 & 0.07 \\
bo7qs3 & 0.9 & yi7qs3 & 0.36 \\
bi8qs3 & -1.4 & yo8qs3 & -1.1 \\
bi9qs3 & -0.35 & yo9qs3 & 0.70 \\
bo10qs3 & -0.65 & yi10qs3 & 1.00 \\
bo11q33 & -0.5 & yi11qs3 & 0.30 \\
bi12qs3 & 0.32 & yo12qs3 & 0.35 \\
bi1qs3 & 0.2 & yo1qs3 & 0.20 \\
bo2qs3 & -1.2 & yi2qs3 & 0.76 \\
bo3qs3 & -0.32 & yi3qs3 & 0.40 \\
bi4qs3 & -0.32 & yo4qs3 & 0.50 \\
\hline \hline
\end{tabular}

\section{CORRECTION METHODS}

There are several ways for the local coupling correction in the RHIC IRs [6]. For example, knowing the triplet roll errors in the one sector in RHIC, it is straightforward to analytically calculate the strengths of the local skew quadrupole correctors based on Eq. (1). The skew quadrupole strength from a normal qudrupole with a roll angle $\theta$ is given by $-2 \theta K_{1}$ when $\theta \ll 1$. Considering the large $\beta \mathrm{s}$ in the IRs, the phase advances in triplets are close to zero. To locally cancel their contributions to the global coupling coefficient $C^{-}$with a local skew quadrupole corrector, we have

$$
\sum_{i=1}^{N} \sqrt{\beta_{x, s} \beta_{y, s}} K_{1 s, s}=0 .
$$

The summation is taken across one sector in RHIC.

With beam, it is possible to localize and to correct the local coupling with IR-bump method [6], action-angle jump [7], and coupling resonance driving term [8]. Among them, IR-bump method is an easy and efficient way to find out the strength for the local skew quadrupole corrector. For this method, we minimize the transverse orbit leakage in one plane from a local orbit bump in another plane by adjusting the strength of local skew quadrupole corrector's strength.

For example, a vertical offset in a skew quadrupole will generate a horizontal closed orbit in the ring,

$$
x_{s}=-\frac{\sqrt{\beta_{x, s} \beta_{x, 0}}}{2 \sin \left(\pi Q_{x}\right)}\left(\Delta y K_{1 s}\right) \cos \left(\pi Q_{x}-\left|\Phi_{x, s}-\Phi_{x, 0}\right|\right) .
$$

To minimize the closed orbit leakage in the horizontal plane from this vertical local bump, we have

$$
\sum_{i=1}^{N} \sqrt{\beta_{x, s}} \Delta y(s) K_{1 s}=0 .
$$




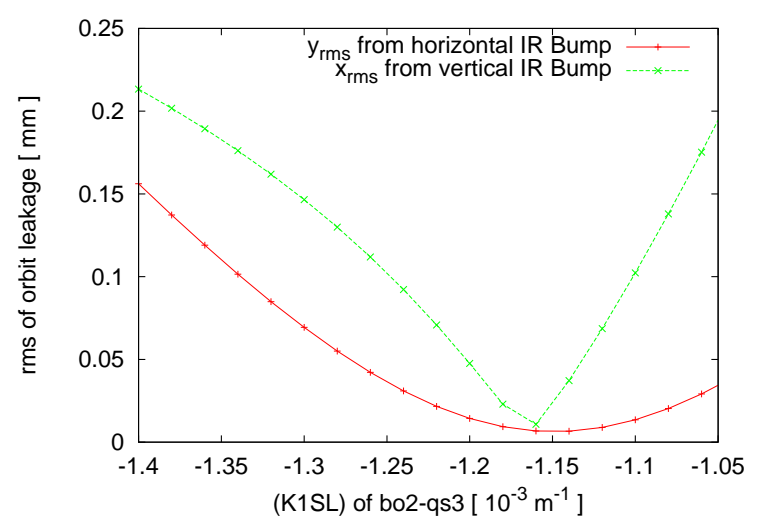

Figure 1: Example of IR-bump method: orbit leakages versus the strength of bo2-qs3.

Here we ignored the phase advances in the IR. The summation is taken cross the local bump. Comparing Eq. (4) with Eq. (2), their weights before $K_{1 s}$ are different. However, if there is no coupling source or coupling sources are completely locally canceled in the bump, there will not be any net orbit leakage in the cross-plane. Both Eq. (2) and Eq. (4) can be used for local coupling correction although they use different observables. Of course, the correction strengths from the two methods may be slightly different.

For RHIC, the available local three bump in one sector will also cover the triplets in the opposite sector, although there the amplitudes of orbits are much smaller. Therefore, the triplet roll error in the opposite sectore also will contribute to orbit leakage in another plane. In the following simulation, we will intentionally block the triplet errors in other sectors in our simulation. If we keep all the measured triplet errors in the simulation, the calculated correction strengths from IR-bump will be more or less different depending on sectors.

In the beam experiment, to achieve a better resoultion in the correction strength scan, the amplitude of local three bump should be large enough to detect the orbit leakage in other plane. And the measurement of rms leakage orbit should have accuracy better than $20 \mu \mathrm{m}$. Normally we will average the local skew quadrupole corrector strengths from the horizontal and vertical bumps. Fig. 1 gives one example of the IR-bump method in a simulation. Here the Blue ring lattice of 2011 RHIC $250 \mathrm{GeV}$ p-p lattice is used. In the simulation, the amplitude of horizontal bump and vertical bump are $18 \mathrm{~mm}$ and $15 \mathrm{~mm}$. By minimizing the rms values of the orbit leakage in another plane, we find the correction strengths for bo2qs3 are $-1.14 \times 10^{-3} \mathrm{~m}^{-1}$ and $-1.16 \times 10^{-3} \mathrm{~m}^{-1}$ with the horizontal and vertical bumps respectively. The averaged correction strength is $-1.15 \times$ $10^{-3} \mathrm{~m}^{-1}$.

\section{CORRECTION AT STORE}

In the following, based on the triplet roll measurements, we will calculate the correction strengths for the local skew quadrupole correctors with the analytical calculation Eq. (2) and the IR-bump method. The correction strengths from them are compared with each other and also compared to the settings in the RHIC control system. Through this study, we would like to find out the differences in the correction strengths between the analytical calculation and the IR-bump method, between injection and store, between the p-p run and the Au-Au run. Since the triplet roll measurements are not available in every sectors, we will only focus on the sectors with the roll measurements.

First we adopt the Blue ring and Yellow ring lattices for the 2011 RHIC $250 \mathrm{GeV}$ p-p run. The $\beta^{*}$ s at IP6 and IP8 are $0.65 \mathrm{~m}$. The $\beta \mathrm{s}$ at other non-colliding IPs are $4 \mathrm{~m}$. Tables 3 and 4 list the correction strengths from the analytical calculation and IR-bump simulation, together with the correction strengths in the control system.

From these tables, the differences in the correction strengths from the horizontal and vertical IR bump are less than $0.1 \times 10^{-3}$. The maximum difference is $0.08 \times 10^{-3}$ for yo5qs3. Comparing the averaged IR-bump correction strengths to the ones with the analytical calculation, the differences are less than $0.1 \times 10^{-3}$ for the Blue ring. In the Yellow ring, except yi2qs3 with $0.12 \times 10^{-3}$ difference, the differences are less than $0.1 \times 10^{-3}$, too.

Then we compare the above correction strengths from simulation calculations with their settings in the online control application. From Table 3, In the Blue ring, the differences between the simulation calculation and the control settings are less than $0.12 \times 10^{-3}$. In the Yellow ring, the differences are less than $0.18 \times 10^{-3}$. The biggest differences between the simulation and the onlione settings are found for bilqs 3 in the Blue ring and yo1qs3 in the Yellow ring.

Table 3: Correction strengths $\left[10^{-3} \mathrm{~m}^{-1}\right]$ with the Blue ring store lattice for the 2011 RHIC $250 \mathrm{GeV}$ p-p runs.

\begin{tabular}{lccccc}
\hline \hline Corr. & Anal. & H-Bump & V-Bump & Avg. & Contr. \\
\hline bi5qs3 & 0.04 & 0.02 & -0.08 & -0.03 & 0 \\
bo6qs3 & 0.04 & 0.02 & 0 & 0.01 & 0.1 \\
bo7qs3 & 0.83 & 0.92 & 0.88 & 0.90 & 0.9 \\
bi8qs3 & -1.45 & -1.40 & -1.48 & -1.44 & -1.4 \\
bi1qs3 & 0.08 & 0.06 & 0.10 & 0.08 & 0.2 \\
bo2qs3 & -1.23 & -1.14 & -1.16 & -1.15 & -1.2 \\
\hline \hline
\end{tabular}

\section{CORRECTION AT INJECTION}

Here we calculate the correction strengths for the local skew quadrupole correctors at injection with the lattices for the 2011 RHIC p-p run. The $\beta^{*}$ s at injection are about $7.5 \mathrm{~m}$ at all IPs. As mentioned above, the correction strengths in the online control application are the same as that at store. Table 5 and Table 6 list the calculation results and the strength settings in the control system. 
Table 4: Correction strengths $\left[10^{-3} \mathrm{~m}^{-1}\right]$ with the Yellow ring store lattice for the 2011 RHIC $250 \mathrm{GeV}$ p-p runs.

\begin{tabular}{lccccc}
\hline \hline Corr. & Anal. & H-Bump & V-Bump & Avg. & Contr. \\
\hline yo5qs3 & -0.21 & -0.15 & -0.18 & -0.17 & -0.26 \\
yi6qs3 & 0.20 & 0.2 & 0.18 & 0.19 & 0.07 \\
yo8qs3 & -1.07 & -1.04 & -1.08 & -1.06 & -1.1 \\
yo1qs3 & 0.02 & -0.09 & 0.03 & -0.03 & 0.2 \\
yi2qs3 & 0.78 & 0.86 & 0.94 & 0.90 & 0.76 \\
\hline \hline
\end{tabular}

Comparing Table 5 and Table 3 for the Blue ring, and Table 6 and Table 4 for the Yellow ring, the difference in the correction strengths from analytical calculation at injection and at store is less than $0.05 \times 10^{-3}$. Then we compare the averaged correction strengths from IR-bump method at injection and store. The differences in the averaged correction strengths from IR-bump methods at injection and at store are less than $0.16 \times 10^{-1} \mathrm{~m}^{-1}$. This bigger difference is from bo2qs3.

Table 5: Correction strengths $\left[10^{-3} \mathrm{~m}^{-1}\right]$ with the Blue ring injection lattice for the 2011 RHIC $250 \mathrm{GeV}$ p-p runs.

\begin{tabular}{lccccc}
\hline \hline Corr. & Anal. & H-Bump & V-Bump & Avg. & Contr. \\
\hline bi5qs3 & 0.03 & 0 & -0.02 & -0.01 & 0 \\
bo6qs3 & 0.08 & 0.04 & -0.04 & 0 & 0.1 \\
bo7qs3 & 0.82 & 0.81 & 0.83 & 0.82 & 0.9 \\
bi8qs3 & -1.45 & -1.40 & -1.51 & -1.46 & -1.4 \\
bi1qs3 & 0.07 & 0.05 & 0.08 & 0.07 & 0.2 \\
bo2qs3 & -1.23 & -1.40 & -1.21 & -1.31 & -1.2 \\
\hline \hline
\end{tabular}

Table 6: Correction strengths $\left[10^{-3} \mathrm{~m}^{-1}\right]$ with the Yellow ring injection lattice for the 2011 RHIC $250 \mathrm{GeV}$ p-p runs.

\begin{tabular}{lccccc}
\hline \hline Corr. & Anal. & H-Bump & V-Bump & Avg. & Contr. \\
\hline yo5qs3 & -0.20 & -0.14 & -0.26 & -0.20 & -0.26 \\
yI6qs3 & 0.19 & 0.19 & 0.17 & 0.18 & 0.07 \\
yo8qs3 & -1.07 & -1.14 & -1.09 & -1.11 & -1.10 \\
yo1qs3 & 0.03 & 0.19 & 0.02 & 0.11 & 0.2 \\
yi2qs3 & 0.79 & 0.87 & 0.95 & 0.91 & 0.76 \\
\hline \hline
\end{tabular}

\section{CORRECTION IN AU-AU RUNS}

Here we calculate the correction strengths for the local skew quadrupoles with the store lattices for the 2011 RHIC $100 \mathrm{GeV}$ Au-Au run, and compare them to the above correction strengths from the lattices for the 2011 RHIC $250 \mathrm{GeV}$ p-p run. The major difference is that we adopt intra-beam scatttering suppression lattices with a bigger phase advances in the arcs for the $\mathrm{Au}-\mathrm{Au}$ run. The integer tunes for the p-p run lattices are $(28,29)$, while for the Au-Au run, they are $(31,32)$. The $\beta^{*}$ s at IP6 and IP8 are $0.7 \mathrm{~m}$ for the Au-Au runs and $0.65 \mathrm{~m}$ for the p-p runs. Table 7 and Table 8 list the calculated local coupling correction strengths based on the triplet roll error measurements.

First we compare the correction strengths from analytical calculation with the store lattices for the $2011 \mathrm{Au}-\mathrm{Au}$ run and the 2011 p-p run. Comparing Table 7 to Table 3, and Table 8 to Table 4 , the correction strengths from the analytical calculation are almost the same with the lattices for the p-p and Au-Au runs. Then we compare the correction strengths from IR-bump methods. The difference in the averaged correction strengths between the p-p and AuAu runs are less $0.15 \times 10^{-3} \mathrm{~m}^{-1}$ for the Blue ring and are less than $0.21 \times 10 \mathrm{~m}^{-1}$ for the Yellow ring. The maximum difference is from yolqs3.

Table 7: Correction strengths $\left[10^{-3} \mathrm{~m}^{-1}\right]$ with the Blue ring store lattice for the 2011 RHIC $100 \mathrm{GeV} \mathrm{Au-Au} \mathrm{runs.}$

\begin{tabular}{lccccc}
\hline \hline Corr. & Anal. & H-Bump & V-Bump & Avg. & Contr. \\
\hline bi5qs3 & 0.04 & 0.02 & -0.02 & 0 & 0 \\
bo6qs3 & 0.04 & 0.02 & -0.02 & 0 & 0.1 \\
bo7qs3 & 0.83 & 0.90 & 0.88 & 0.89 & 0.9 \\
bi8qs3 & -1.45 & -1.40 & -1.74 & -1.57 & -1.4 \\
bi1qs3 & 0.07 & 0.06 & 0.10 & 0.08 & 0.2 \\
bo2qs3 & -1.24 & -1.16 & -1.18 & -1.17 & -1.2 \\
\hline \hline
\end{tabular}

Table 8: Correction strengths $\left[10^{-3} \mathrm{~m}^{-1}\right]$ with the Yellow store lattice for the 2011 RHIC $100 \mathrm{GeV}$ Au-Au runs

\begin{tabular}{lccccc}
\hline \hline Corr. & Anal. & H-Bump & V-Bump & Avg. & Contr. \\
\hline yo5qs3 & -0.21 & -0.30 & -0.27 & -0.29 & -0.26 \\
yi6qs3 & 0.20 & 0.20 & 0.30 & 0.25 & 0.07 \\
yo8qs3 & -1.07 & -1.30 & -1.11 & -1.21 & -1.1 \\
yo1qs3 & 0.02 & -0.18 & -0.29 & -0.24 & 0.2 \\
yi2qs3 & 0.79 & 0.88 & 0.98 & 0.93 & 0.76 \\
\hline \hline
\end{tabular}

\section{$C^{-}$AFTER IR-BUMP CORRECTION}

As we know, the local coupling correction with the IRbump method will reduce the orbit cross-talk between the horizontal and vertical planes. Here we check if the local coupling correction is good enough for the global coupling correction. For the global coupling correction, we require the global coupling coefficient or $\Delta Q_{\min }$ should be smaller than $10^{-4}$ after correction. As we discussed earlier, the IR-bump method focuses on correcting the crossplane orbit leakage from a local orbit bump. The correction strengths may be slightly different from the analytical calculation based on Eq.(2). 
Table 9 shows that the contributions to the global coupling coefficient from each sector. The triplet roll errors in Table 1 are used. The strengths of the local skew quadrupole correctors are the averaged ones from the IRbump method and their settings from the online application listed in Table 3. In this study, the store lattice of the Blue ring lattice in the $2011 \mathrm{p}-\mathrm{p}$ run is used.

From Table 9, without local coupling correction, the coupling coefficient $C^{-}$or $\Delta Q_{\min }$ contributed from the triplet rolls in the sectors 7,8 , and 2 are about $0.0587,0.1865$, and 0.3273 . The contributions from sectors 5,6 , and 1 are below 0.01 . As we mentioned above, the triplet roll errors in the sectors 5 and 6 had been adjusted to minimize their contributions to the global coupling. From this calculation, the local coupling corrections in the sectors 7,8 , and 2 are required to reduce their contributions to $\Delta Q_{\min }$.

With the averaged correction strengths from the IRbump simulations, the global coupling coefficient contributions from the triplets are reduced, especially for the sectors 7,8 , and 2 . The maximum contribution is about 0.016 from the sector 7. With the current strengths settings of local skew quadrupole correctors from the online application, the maximum contribution to the global coupling coefficient is about 0.017 from the same sector. To achieve $\Delta Q_{\min }$ below $10^{-4}$, the global coupling feedback with the global skew quadrupoles is necessary.

Table 9: $C^{-}$without and with local coupling corrections. The correction strengths of local skew quadrupole correctors are the averaged ones from the IR-bump method and their settings from the online application.

\begin{tabular}{lccc}
\hline \hline Sector & Triplets & IR-bump Corr. & Online Corr. \\
5 & 0.0086 & 0.0153 & 0.0086 \\
6 & 0.0098 & 0.0076 & 0.0127 \\
7 & 0.1865 & 0.016 & 0.0167 \\
8 & 0.3273 & 0.0025 & 0.0115 \\
1 & 0.0037 & $5.5 \times 10^{-5}$ & 0.0057 \\
2 & 0.0587 & 0.0038 & 0.0014 \\
\hline \hline
\end{tabular}

\section{VERTICAL DISPERSION CONCERNS}

In this section we calculate the vertical dispersion from triplet rolls and local and global skew quadrupoles. In the $2011 \mathrm{p}$-p run a vertical dispersion with a maximum of $0.5 \mathrm{~m}$ was measured in RHIC. Vertical dispersion may cause depolarization on the energy acceleration and at physics store. In the following calculation, we will adopt the Blue ring store lattice for the 2011 RHIC $250 \mathrm{GeV}$ run.

The dispersion leakage by a skew quadrupole with a strength $K_{1 s, 0}$ is given by

$$
\begin{aligned}
D_{y, s}=\quad & -\frac{\sqrt{\beta_{y, s} \beta_{y, 0}}}{2 \sin \left(\pi Q_{y}\right)}\left(D_{x, 0} K_{1 s, 0}\right) \\
& \cos \left(\pi Q_{y}-\left|\Phi_{y, s}-\Phi_{y, 0}\right|\right) .
\end{aligned}
$$

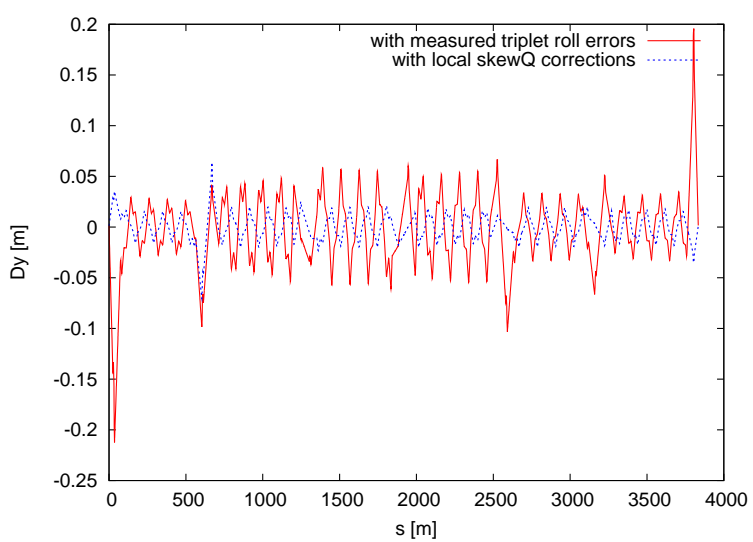

Figure 2: Calculated vertical dispersion from the measured triplet roll errors and with local coupling correction.

Here $D_{x, 0}$ is the horizontal dispersion at the skew quadrupole. Ignoring the phase advances in the triplets, to completely cancel the vertical dispersion leakage, we obtain

$$
\sum_{i=1}^{N} \sqrt{\beta_{y}} D_{x} K_{1 s}=0 .
$$

The scaling factor in Eq. (6) for the global dispersion cancellation is different from Eq. (2) for the global coupling correction and from Eq. (4) for the orbit leakage cancellation. However, if there is no coupling source or all coupling sources are fully compensated locally in this sector, the vertical dispersion leakage should be zero. Again, Eqs. (2), (4) and (6) can be used for local coupling correction although they use different observables. The correction strengths from Eqs. (2), (4) and (6) may be slightly different.

Figure 2 shows the vertical dispersion with the triplet rolls and their local skew quadrupole corrections in the sectors where the measurement data of triplet rolls are available. From it, the triplet rolls introduce a vertical dispersion with a maximum of $0.2 \mathrm{~m}$ in the IRs if they are not corrected. The reason is that the $\beta_{y}$ is big and the horizontal dispersion $D_{x}$ is not zero in IRs. After the local coupling correction with the strengths found with the above analytical calculation or IR-bump method, the vertical dispersion is greatly reduced and is tolerable.

Figure 3 shows the vertical dispersion from the triplet roll errors, the local and global skew quadrupoles from the control system. From it, the online global skew quadrupoles for the global coupling correction introduce a vertical dispersion with a maximum of $0.45 \mathrm{~m}$. Therefore it is important not to introduce additional vertical dispersion by the global coupling correction.

For comparison, we simulate the vertical dispersion solely from vertical dipole kickers. The vertical dispersion from vertical dipole kickers is mainly from the vertical closed orbits in the arc sextupoles. A vertical offset of the beam in the normal sextupole will generate a skew quadrupole feed-down. As an example, we generate a closed orbit with random strength assignments to all the 


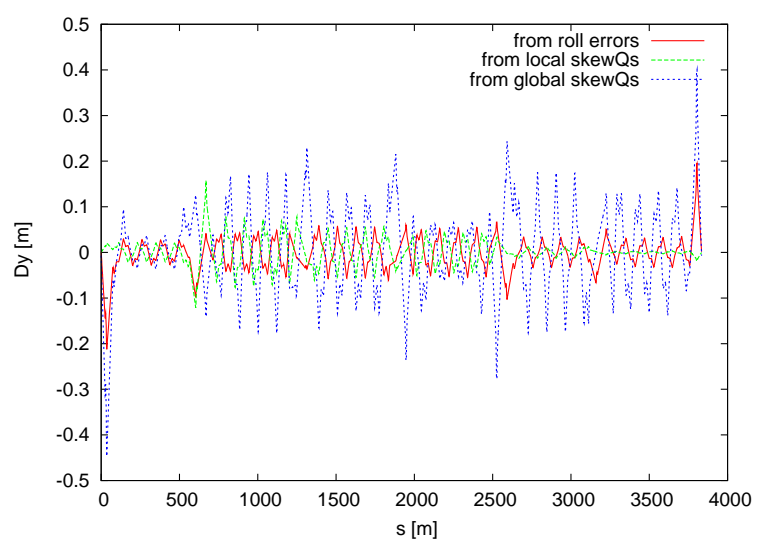

Figure 3: Vertical disperison with measured triplet roll errors and the local and global skew quadrupoles from the online application.

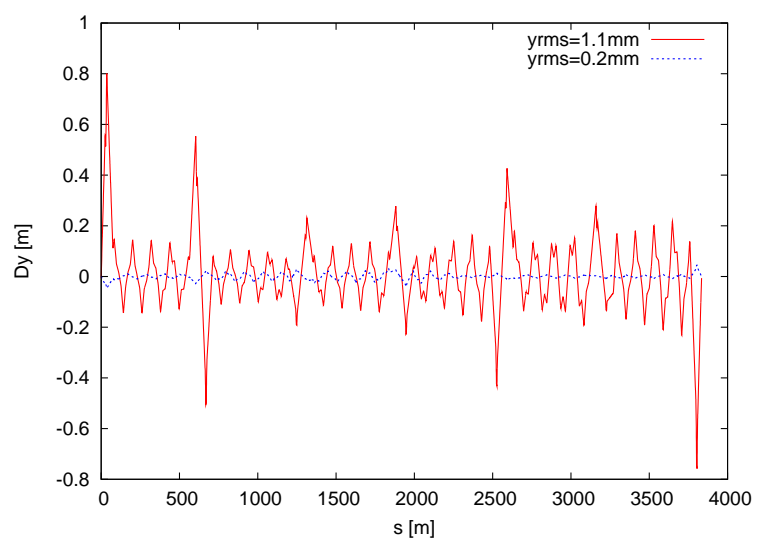

Figure 4: Vertical disperison generated by vertical obits with $y_{r m s}=1 \mathrm{~mm}$ and $y_{r m s}=0.2 \mathrm{~mm}$.

vertical dipole kickers. The rms values of vertical closed orbit is about $1.1 \mathrm{~mm}$ without orbit correction. Then we correct it with sliding bumps to achieve $y_{r m s} 0.2 \mathrm{~mm}$. Figure 4 shows the vertical dispersion with $y_{r m s}=1 \mathrm{~mm}$ and $y_{r m s}=0.2 \mathrm{~mm}$ in this example. From it, a vertical closed orbit with $y_{r m s}=1 \mathrm{~mm}$ will introduce a vertical dispersion with a maximum of $0.8 \mathrm{~m}$ in the IRs. With $y_{r m s}=0.2 \mathrm{~mm}$, the vertical dispersion is negligible.

\section{SUMMARY}

In the article, based on the measured triplet roll errors in 2001 and 2004, we calculated and compared the local coupling correction strengths with analytical calculation and IR-bnump method. The correction strengths from these two methods agree very well except one corrector. And through simulation, we found that the local skew quadrupole correction strengths vary less than $0.21 \times 10^{-1}$ at injection, at store and for the p-p and Au-Au runs. After the local coupling correction with the IR-bump method, the cross-talk of the transverse orbits and the vertical dispersion are reduced. However, to reduce $\Delta Q_{\min }$ to below
$10^{-4}$, the global coupling feedback with the global skew quadrupoles is necessary. We also calculated the vertical dispersion contributed by the triplet rolls, their local corrections, and the global skew quadrupoles from the control system. We found that a perfect local coupling correction reduces the vertical dispersion leakage. The online global coupling correction quadrupoles introduce an additional vertical dispersion with a maximum of $0.5 \mathrm{~m}$. We are planning to construct a global coupling feedback which more skew quadrupole families to correct the global coupling and to reduce its contribution to the vertical dispersion [3].

\section{REFERENCES}

[1] Y. Luo, et al., Phys. Rev. ST Accel. Beams 9, 124001 (2006).

[2] P. Cameron, et al., Phys. Rev. ST Accel. Beams 9, 122801 (2006).

[3] C. Liu, et al., BNL C-AD AP Note ???, 2011.

[4] V. Ptitsyn, private communication, 2011.

[5] Y. Luo, BNL C-AD AP Note 388, 2010.

[6] F. Pilat, et al, BNL C-AD AP Note 77, 2002.

[7] J. Cardona, et al., Phys. Rev. ST Accel. Beams 12, 014002 (2009).

[8] R. Calaga, et al., Phys. Rev. ST Accel. Beams 8, 034001 (2005). 